\title{
Neutrophil extracellular traps in physiology and pathology
}

\author{
ANETA MANDA, MICHAE P. PRUCHNIAK, MAGDALENA ARAŹNA, URSZULA A. DEMKOW
}

Medical University of Warsaw, Poland

\begin{abstract}
Neutrophil extracellular traps (NETs) are developed by nature to protect the body from furious invaders. On the other hand NETs can play an important role in human pathology. Recent studies have shown that neutrophils are able to perform beneficial suicide to create an unique microbicidal net composed from cellular content attached to chromatic frame. It is a powerful tool that primary serve as protector from severe infections, but this weapon is also a double ended sword of the immunity. If overproduced NETs provoke certain autoimmune diseases, coagulation disorders and even cancer metastases. Moreover, due to the competition between host and pathogens, the microorganism have developed a width repertoire of sophisticated evading mechanisms, like creation of polysaccharide capsule or changes in cell wall charge. Therefore it is important to increase the knowledge about paths underlying NETs formation and degradation processes if we want to efficiently fight with bacterial infections and certain diseases.
\end{abstract}

Key words: neutrophils extracellular traps, NET in physiology and pathology, bacterial infections.

(Centr Eur J Immunol 2014; 39 (1): 116-121)

\section{Introduction}

Polymorphonuclear leukocytes (PMNs), or neutrophils, are most abundant population of white blood cells, comprising $50-75 \%$ of leukocytes in peripheral blood. They serve as the first line of innate immune defense against pathogens. These cells employ several strategies to fight infections, two of which have been well established and described: phagocytosis and degranulation, which releases lytic enzymes. They are also characterized by their ability to form reactive oxygen species (ROS) by NADPH oxidase which have antimicrobial potential [1]. In 2004 a novel mechanism that combats infections was described. Neutrophils can form extracellular web-like structures, called neutrophil extracellular traps (NETs), made up of decondensed chromatin associated with antimicrobial proteins and peptides [2]. Since this discovery a lot of studies have been performed to evaluate the formation process and physiological role of NETs in defense against invading pathogens. This unique structure is considered to play a role not only in host defense mechanisms, but also to have unfavorable consequences when the balance between NETs formation and clearance is disturbed. Thus, a significant field of NET research is centered on the relationship between NETs and inflammation, infection or autoimmune diseases [3]. In this review, we will present current knowledge about this sophisticated relationship and the role of NETs in infections as well as in certain diseases.

\section{Neutrophil extracellular traps}

Upon activation, the chromatin in neutrophils starts to decondensate and forms complexes with over 30 granular and cytoplasmic proteins, which are finally extruded into the extracellular space. Polymorphonuclear leukocytes not only act defensively in the bloodstream but also may cover large areas within the solid tissues when crawling. Interestingly, at most $20-25 \%$ of these residual cells release NETs to fight threats $[2,4]$.

Neutrophil extracellular traps were primarily observed as fragile fibers composed of smooth stretches with globular domains, which aggregated into larger strands. Further examinations showed that NETs structures may be degraded by treatment with DNases and not by proteases, therefore nuclear DNA (chromatin) is certain to be the backbone of NETs structure [2,5]. This bioframe is ornamented with a number of protein components which stabilize this structure as well show microbicidal properties (Table 1) [6].

The role of extracellular traps is to create a specific space with high local concentration of antimicrobial agents, preferably at the site of infection. They simply immobilize and kill microorganisms preventing them from dissemination. Neutrophil extracellular traps formation can be triggered by a broad range of stimulators. Pathogens, their products, as well as variety of biochemical agents, i.e. phorbol-12-myristate-13-acetate (PMA), lipopolysaccharide (LPS), interleukin-8 (IL-8), tumor necrosis factor $\alpha$ 
Table 1. Main neutrophil derived proteins which ornaments neutrophils extracellular trap structure

\begin{tabular}{ll}
\hline \multicolumn{1}{c}{ Origin } & \multicolumn{1}{c}{ Neutrophils extracellular trap's proteins } \\
\hline histones & Type of protein \\
\hline primary (azurophilic) granules & $\begin{array}{l}\text { neutophil elastase (NE), myeloperoxidase (MPO), cathepsin G, defensins (HNP-1, HNP-2 and HNP-3 } \\
\text { peptides), bacterial permeability increasing protein (BPI) }\end{array}$ \\
\hline secondary (specific) granules & alkaline phosphatase, lactoferrin, lysozyme, NADPH oxidase, cathelicidin and collagenase \\
\hline tertiary granules & cathepsin, gelatinase [also known as metalloprotease 9 (MMP9)] \\
\hline cytosol & proteinase 3 (PR3), LL-37, tryptase, neutophil serine protease 4 (NSP4) \\
\hline
\end{tabular}

(TNF- $\alpha$ ), monosodium urate crystals, nitric oxide (NO), autoantibodies and immune complexes can initiate traps formation. Moreover, interaction between neutrophils and platelets via Toll-like-receptor 4 (TLR4) leads to the formation of NETs in septic blood [3, 7]. Recent studies have shown that macrophages are responsible for NETs clearing after they execute their function. This path starts when DNAse I breaks extracellular traps structure and the complement factor $\mathrm{C} 1 \mathrm{q}$ opsonizes the debris. Both processes facilitate the clearance of web-like structures in vivo. Subsequently NETs are internalized by macrophages due to cytochalasin D-dependent endocytic process. Actively engulfed NETs are apparently degraded in lysosomes, but it is possible that other intracellular compartments are also involved [8].

Polymorphonuclear leukocytes are not the only cells able to form extracellular traps. Several studies have shown that mast cells and macrophages are also prone to create similar structures in response to pathogens and certain stimulatory agents. Interestingly, extracellular traps released by eosinophils are made of mitochondrial DNA, therefore they lack histones, furthermore this process never causes cell death [9].

\section{Trap formation}

To date, two mechanisms of NETs formation are known. The major route, a slow lytic cell death mechanism, is also known as suicidal Netosis. The second strategy, called vital Netosis, enables cells to continue their functions, such as crawling and phagocytosis after NET release. Netosis via cell death occurs 2-4 hours after stimulation, whereas vital Netosis occurs rapidly, approximately 5-60 minutes after stimulation. Toll-like receptor 2 (TLR2) and complement factor 3 contribute to both suicidal and vital Netosis, but are not sufficient to stimulate NETs formation in isolated PMNs [3, 4].

When PMN undergoes suicidal Netosis, its nuclear and granule membranes disassemble and decondensation of chromatin occurs. This process is continued in the cy- tosol, where granular and cytoplasmic proteins associate with DNA and histones. Neutrophil extracellular traps are released after the plasma membrane rupture. This process had been initially considered as dependent on ROS and NADPH oxidase. This hypothesis was supported by following findings: PMNs of Nox2-deficient (Nox2 is a subunit of NADPH) mice could not form NETs after the stimulation with PMA, just as neutrophils from patients suffering from chronic granulomatous disease where Nox2 is defective or absent $[1,10,11]$. Reactive oxygen species participate in NETs formation, but the exact molecular mechanism is yet poorly understood. According to reported data, ROS induce the translocation of neutrophil elastase from the primary granules to the nucleus, where it degrades histones and causes chromatin decondensation. Afterwards, MPO acts in synergy with NE to drive further relaxation of chromatin. The above mechanism is not dependent on MPO enzymatic activity [11]. Nevertheless, it has been described that it is possible to release NETs via cell death mechanism independently from ROS. Polymorphonuclear leukocytes stimulation with Staphylococcus aureus culture may release NETs before any ROS can be detected. Thus, the ROS participate only in the production of certain type of extracellular traps [12, 13].

Another process that is important but not sufficient for Netosis is citrullination of histones. There are five known isoforms of peptidylarginine deiminase (PAD), of which only PAD4 can be translocated to the nucleus and catalyse deamination of arginine to citrulline. Inhibition of PAD4 with C1-amidine disrupts NETs formation. However, PMA as a strong inducer of Netosis, is simultaneously an activator of protein kinase $\mathrm{C}$ (PKC), an inhibitor of PAD4. These observations indicate that there is no absolute connection between NETs formation and citrullination of histones and other regulating mechanisms are also involved $[14,15]$.

Some scientists suggest that trap formation is also connected with autophagy. The addition of wortmannin, pharmacological inhibitor of autophagy, impairs chromatin decondensation, but does not affect NADPH oxidase-dependent oxidative burst [12]. 
It is worth noting, that suicidal Netosis is different from necrosis and apoptosis. During Netosis there is no DNA fragmentation and caspases appear not to be involved, what distinguish this process from apoptosis. Moreover morphological features of dying cell are different in both processes. Additionally in early necrosis there are no morphological changes in the nucleus, whereas NETs release is preceded by nuclear membranes disintegration and formation of vesicles [12].

Vital Netosis is a rapid process, undertaken by a small subset of neutrophils. The process starts from the separation of the nuclear membranes, followed by decondensation of chromatin in the intermembrane space. Vesicles containing chromatin and antimicrobial proteins are formed and they accumulate near to the plasma membrane. Finally vesicles release their content into the extracellular space, where proteins and chromatin assemble into NETs [4]. It has been documented that there is another process enabling NETs formation - the release of mitochondrial DNA. Anuclear PMNs, cytoplasts or motile cytokineplasts, maintain the ability to chemotax and engulf bacteria in the bloodstream. The fact that PMNs can function without nucleus is not unlikely as it has been reported that neutrophils may express the same protein (survivine) as erythrocytes, another anucleated cells. Survivine is an inhibitor of apoptosis, its overexpression induces enucleation of differentiating erythroblasts. Mature PMNs are able to synthesize this protein after stimulation with macrophage-colony-stimulating factor [16-19].

\section{Neutrophil extracellular traps in infection and autoimmune diseases}

Neutrophil extracellular traps are being formed to trap and kill a variety of pathogens including bacteria, fungi, viruses and parasites. They can prevent infection from spreading and ensure high local concentrations of antimicrobial factors. Considering available scientific data, it seems that NETs formation occurs in response to specific microbes, but properties of pathogens that are necessary to induce Netosis remain to be further investigated [4].

Neutrophil extracellular traps play a vital role in the defense against wide range of bacteria. Both Gram-positive and Gram-negative microbes may induce NETs formation. It was proven that Netosis occurs after stimulation with pathogens such as Streptococcus pneumoniae, Streptococcus pyogenes, Staphylococcus aureus, Escherichia coli, Salmonella typhimurium or Shigella flexnerii. Besides trapping and killing bacteria, active components of extracellular traps can neutralize virulence factors. As an example invasion plasmid antigens: IpaB and IcsA (used for cell-to-cell spread) from $S$. flexnerii, are degraded by neutrophil elastase (NE) which is essential part of NETs [2, $20,21]$. Moreover, it has been indicated that some strains of Mycobacterium tuberculosis and Mycobacterium canettii, might trigger NET formation. Neutrophil extracellular trap prevents dissemination of $M$. tuberculosis bacilli but does not kill them [22].

There are some strategies developed by bacteria to escape from extracellular traps killing mechanism. As an example $S$. pneumoniae has polysaccharide capsule that reduces NET binding. It can also modify lipoteichoic acids in cellular wall by activation of dlt operon. Subsequently the surface charge of cell turns from negative into positive, disrupting its affinity to antibacterial factors [23]. $S$. pyogenes produces Dnase Sda1 and virulence factor M1 neutralizing cathelicidin, the antimicrobial component of NET $[22,24]$.

Extracellular traps can act not only within the bloodstream and tissues. It has been reported that NETs may help to avoid spreading of bacterial biofilm coating the dental root in patients with periodontitis. On the one hand, the bacterial proliferation is prevented by overproduction of the gingival cervical fluid, around the tooth necks, containing neutrophils producing extracellular traps. On the other hand, periodontal bacteria show some resistance to NETs. Palmer et al. showed that biofilm in periodontitis is prone to efficient production of Dnases which break the trap's bioframe. In normal conditions the balance between biofilm degradation and bacterial protection is established. But when balance is moved in favor of bacteria a periodontitis easily occurs [25].

These unique biological weapon is also involved in fighting with fungal infections. Urban et al. showed that the antimicrobial heterodimer calprotectin is the major antifungal component of extracellular traps. These authors showed that calprotectin deficient mice are highly prone to candidiasis and aspergillosis thus, they postulated that this protein appears to be one of main factors involved in fungal clearance [26, 27].

It is unresolved why extracellular trap are more beneficial during fungal infection than phagocytosis or degranulation. Probably fungi are simply too large to be internalised by phagocytes. It was shown that invasive forms of C. albicans are being effectively killed within trap structures rather than with use of any other neutrophil's weapons. This was strictly connected with the fact that hyphae are too big for effective phagocytosis [5]. Bruns et al. showed that Aspergillus fumigatus hyphae are stronger inducers of NETs than conidia. This alteration of host-pathogen interaction dynamics is explained by presence of hydrophobin RodA. This protein is expressed only upon the surface of conidia and makes conidia immunologically inert, thus leading to reduced NET formation [28].

The protein based arsenal incorporated within trap backbone is enormous. Among those proteins there are some with antiviral properties, i.e. myeloperoxidase and $\alpha$-defensin. Thus, destruction of viruses by neutrophils is possible. Saitoh et al. postulated that Human Immunode- 
ficiency Virus-1 can induce traps formation via suicidal pathway. They showed that neutrophils detect HIV-1 via two Toll-like receptors (TLRs): TLR7 and TLR8, which recognize viral nucleic acids. Engagement of TLR7 and TLR8 induces the intracellular cascade of events which in the end promote generation of reactive oxygen species that trigger NET formation, leading to NET-dependent HIV-1 elimination. HIV virus tries to avoid entrapment using a unique sophisticated mechanism. HIV-1 counteracts this defensive response by inducing C-type lectin CD209-dependent production of interleukin (IL)-10 by dendritic cells in order to inhibit NET formation. Overproduction of IL-10 suppresses the reactive oxygen species-dependent generation of NETs induced upon TLR7 and TLR8 engagement, resulting in disrupted NET-dependent HIV-1 elimination [29, 30].

Another virus that have been described to initiate $\mathrm{Ne}$ tosis is influenza virus which causes acute respiratory distress syndrome (ARDS). Studies showed that Netosis may occur after activation of lung epithelium. Administration of anti-myeloperoxidase antibody and an inhibitor of superoxide dismutase prevent traps from being formed. This observation indicates that NETs generation is induced by redox enzymes during influenza infection and superoxide and $\mathrm{H}_{2} \mathrm{O}_{2}$ produced by epithelium are mediators in this process [31].

The potential role of NETs in parasites elimination was also considered. Some scientists showed that NET forms a space that physically contain the pathogen but does not kill it. The precise mechanism underlying this host-threat interaction is yet poorly understood. The production of NETs has been observed after stimulation with Leishmania spp. (inter alia Lamozonensis) [32], Eimeria bovis [33] or Toxoplasma gondii [34]. Neutrophil extracellular traps have been also found in peripheral blood of patiens infected with Plasmodium falciparum [35].

Besides these benefits, there are a number of studies pointing that Netosis is a double-edge sword of the immunity. Despite the beneficial role of preventing infectious threats NETs formation participates in the pathogenesis of many diseases, including inflammatory and autoimmune conditions. It is believed that Netosis has unfavorable consequences when happens in the inappropriate localization, with the inadequate intensity or in the wrong time.

To date, Netosis is considered as a process causing lung epithelium cells damage in patients with cystic fibrosis (CF). Cystic fibrosis patients suffer from chronic lung infection and inflammation due to the secretion of viscous sputum. Sputum viscosity is caused by extracellular DNA. In the past, the extracellular DNA found in CF sputum was thought to originate from dying necrotic or apoptotic neutrophils and epithelial cells. However, some studies showed that in that case, the chromatin would remain condensed and would not be found in complex with neutrophil granular proteins. Thus, it was proposed that genomic DNA ornamented by neutrophil elastase (NE) and MPO, have the most detrimental effect on lung tissue [36].

Data indicate possible relationship between preeclampsia and NETs formation exists. Neutrophils extracellular traps have been found in the intervillous space of preeclamptic placentae. The elevated level of extracellular DNA seems to be caused by the placentae derived factors, i.e. IL-8 and placental micro-debris. There is a straight connection between NETs generation and disease pathogenesis because preeclampsia episodes coexist with an increased levels of cell-free DNA in plasma. However, the exact relation between Netosis and preeclampsia remains to be explained [37].

As mentioned above, PMNs - platelets interaction during sepsis triggers NETs release. The circulating trap complexes can damage the endothelium. Histones bind to endothelium surface where they promote platelets aggregation and platelet-dependent thrombin generation. Simultaneously, due to the presence of extracellular chromatin fibers, scaffolds in circulation are being formed. These events lead to thrombus formation and cause disturbances in blood flow. These findings suggest, that Netosis may sharply increase the risk of deep vein thrombosis [38].

Contradicting results have been observed when studying the role of Netosis in cancer and this area needs to be further investigated. Some studies show that cancer patients who suffer from severe postoperative infections are more likely to die from metastasis than those who do not. There is a link between metastases, infections and extracellular traps. It was proposed that NETs may trap circulating tumor cells and enhance their adhesion to distant organ sites, thus promote metastasis. Nonetheless, neutrophil elastase, one of the main components of traps, seems to have ability to enhance tumor-cell proliferation [39].

Release of extracellular traps contribute to augment the autoimmune response. Many studies have been exploring this area. NETs formation is involved at least in pathogenesis of ANCA vasculitis, systemic lupus erythematosus (SLE) and rheumatoid arthritis [3, 40, 41]. Neutrophil extracellular trap itself is an autological structure and it persistent existence can cause overproduction of autoantibodies against DNA, RNA and specific proteins. It is postulated that NET takes a prominent role during exacerbations of those diseases.

There are at least two pathways by which NET proteins could have impact on SLE pathogenesis. Neutrophil extracellular trap may contribute to organs damage, due to the potential toxicity of NET's ornaments. To prove this theory typical trap associated proteins were quantified. In a result they have been detected at increased levels in SLE patients compared with healthy ones. The second concept is that NET proteins may be specifically modified and positioned to break tolerance and thereby trigger or exacerbate autoimmunity. Idea that modified proteins serve as autoantigens in SLE is known for some time but attempts to link them to extracellular traps are new. 
Studies showed that NET-proteins like C1q, NE, histones, HMGB1 (high-mobility group protein B1), HNP (human neutrophil peptide 1), IL-17, IL37, cathelicidin and MPO contribute to SLE pathogenesis [42].

\section{Summary}

The double edged sword of the immunity, as extracellular traps are called, can be both beneficial and threatful. Neutrophils for many years were considered to be guardians fighting with prokaryotic and eukaryotic invaders. There is increasing evidence that NETs play an important role in several infectious as well as noninfectious diseases. Since the discovery of Netosis neutrophils started to be considered as play prominent players in autoimmunity, inflammatory states and thrombosis.

\section{Authors declare no conflict of interest.}

\section{References}

1. Mantovani A, Cassatella MA, Costantini C, Jaillon S (2011): Neutrophils in the activation and regulation of innate and adaptive immunity. Nat Rev Immunol 11: 519-531.

2. Brinkmann V, Reichard U, Goosmann C, et al. (2004): Neutrophil extracellular traps kill bacteria. Science 303: 1532-1535.

3. Yu Y, Su K (2013): Neutrophil extracellular traps and systemic lupus erythematosus. J Clin Cell Immunol 4.

4. Branzk N, Papayannopoulos V (2013): Molecular mechanisms regulating NETosis in infection and disease. Semin Immunopathol 35: 513-530.

5. Urban CF, Reichard U, Brinkmann V, Zychlinsky A (2006): Neutrophil extracellular traps capture and kill Candida albicans yeast and hyphal forms. Cell Microbiol 8: 668-676.

6. Pruchniak MP, Arazna M, Demkow U (2013): Life of neutrophil: from stem cell to neutrophil extracellular trap. Respir Physiol Neurobiol 187: 68-73.

7. Clark SR, Ma AC, Tavener SA, et al. (2007): Platelet TLR4 activates neutrophil extracellular traps to ensnare bacteria in septic blood. Nat Med 13: 463-469.

8. Farrera C, Fadeel B (2013): Macrophage clearance of neutrophil extracellular traps is a silent process. J Immunol 191: 2647-2656.

9. von Köckritz-Blickwede M, Goldmann O, Thulin P, et al. (2008): Phagocytosis-independent antimicrobial activity of mast cells by means of extracellular trap formation. Blood 111: 3070-3080.

10. Campbell AM, Kashgarian M, Shlomchik MJ (2012): NADPH oxidase inhibits the pathogenesis of systemic lupus erythematosus. Sci Transl Med 4: 157ra141.

11. Fuchs TA, Abed U, Goosmann C, et al. (2007): Novel cell death program leads to neutrophil extracellular traps. J Cell Biol 176: 231-241.

12. Remijsen Q, Vanden Berghe T, Wirawan E, et al. (2011): Neutrophil extracellular trap cell death requires both autophagy and superoxide generation. Cell Res 21: 290-304.

13. Pilsczek FH, Salina D, Poon KK, et al. (2010): A novel mechanism of rapid nuclear neutrophil extracellular trap formation in response to Staphylococcus aureus. J Immunol 185: 74137425.

14. Wang Y, Li M, Stadler S, et al. (2009): Histone hypercitrullination mediates chromatin decondensation and neutrophil extracellular trap formation. J Cell Biol 184: 205-213.

15. Neeli I, Radic M (2013): Opposition between PKC isoforms regulates histone deimination and neutrophil extracellular chromatin release. Front Immunol 4: 38.

16. Yousefi S, Mihalache C, Kozlowski E, et al. (2009): Viable neutrophils release mitochondrial DNA to form neutrophil extracellular traps. Cell Death Differ 16: 1438-1444.

17. Yipp BG, Petri B, Salina D, et al. (2012): Infection-induced NETosis is a dynamic process involving neutrophil multitasking in vivo. Nat Med 18: 1386-1393.

18. Altznauer F, Martinelli S, Yousefi S, et al. (2004): Inflammation-associated cell cycle-independent block of apoptosis by survivin in terminally differentiated neutrophils. J Exp Med 199: 1343-1354.

19. Keerthivasan G, Liu H, Gump JM, et al. (2012): A novel role for survivin in erythroblast enucleation. Haematologica 97: 1471-1479.

20. Wartha F, Beiter K, Albiger B, et al. (2007): Capsule and D-alanylated lipoteichoic acids protect Streptococcus pneumoniae against neutrophil extracellular traps. Cell Microbiol 9: 1162-1171.

21. Lauth X, von Köckritz-Blickwede M, McNamara CW, et al. (2009): M1 protein allows Group A streptococcal survival in phagocyte extracellular traps through cathelicidin inhibition. J Innate Immun 1: 202-214.

22. Ramos-Kichik V, Mondragón-Flores R, Mondragón-Castelán M, et al. (2009): Neutrophil extracellular traps are induced by Mycobacterium tuberculosis. Tuberculosis (Edinb) 89: 29-37.

23. Marin-Esteban V, Turbica I, Dufour G, et al. (2012): Afa/ Dr diffusely adhering Escherichia coli strain C1845 induces neutrophil extracellular traps that kill bacteria and damage human enterocyte-like cells. Infect Immun 80: 1891-1899.

24. Vitkov L, Klappacher M, Hannig M, Krautgartner WD (2009): Extracellular neutrophil traps in periodontitis. J Periodontal Res 44: 664-672.

25. Palmer LJ, Chapple IL, Wright HJ, et al. (2012): Extracellular deoxyribonuclease production by periodontal bacteria. J Periodontal Res 47: 439-445.

26. Urban CF, Ermert D, Schmid M, et al. (2009): Neutrophil extracellular traps contain calprotectin, a cytosolic protein complex involved in host defense against Candida albicans. PLoS Pathog 5: e1000639.

27. Bianchi M, Niemiec MJ, Siler U, et al. (2011): Restoration of anti-Aspergillus defense by neutrophil extracellular traps in human chronic granulomatous disease after gene therapy is calprotectin-dependent. J Allergy Clin Immunol 127: 1243$1252 \mathrm{e} 7$.

28. Bruns S, Kniemeyer O, Hasenberg M, et al. (2010): Production of extracellular traps against Aspergillus fumigatus in vitro and in infected lung tissue is dependent on invading neutrophils and influenced by hydrophobin RodA. PLoS Pathog 6: e1000873.

29. Saitoh T, Komano J, Saitoh Y, et al. (2012): Neutrophil extracellular traps mediate a host defense response to human immunodeficiency virus-1. Cell Host Microbe 12: 109-116.

30. Dabrowska A, Slotwinski R, Kedziora S (2012): Inflammation and cancer - double face of Toll-like receptors. Centr Eur J Immunol 37: 67-70. 
31. Narasaraju T, Yang E, Samy RP, et al. (2011): Excessive neutrophils and neutrophil extracellular traps contribute to acute lung injury of influenza pneumonitis. Am J Pathol 179: 199210.

32. Guimaraes-Costa AB, Nascimento MT, Froment GS, et al. (2009): Leishmania amazonensis promastigotes induce and are killed by neutrophil extracellular traps. Proc Natl Acad Sci U S A 106: 6748-6753.

33. Behrendt JH, Ruiz A, Zahner H, et al. (2010): Neutrophil extracellular trap formation as innate immune reactions against the apicomplexan parasite Eimeria bovis. Vet Immunol Immunopathol 133: 1-8.

34. Abi Abdallah DS, Lin C, Ball CJ, et al. (2012): Toxoplasma gondii triggers release of human and mouse neutrophil extracellular traps. Infect Immun 80: 768-777.

35. Baker VS, Imade GE, Molta NB, et al. (2008): Cytokine-associated neutrophil extracellular traps and antinuclear antibodies in Plasmodium falciparum infected children under six years of age. Malar J 7: 41.

36. Papayannopoulos V, Staab D, Zychlinsky A (2011): Neutrophil elastase enhances sputum solubilization in cystic fibrosis patients receiving DNase therapy. PLoS One 6: e28526.

37. Gupta AK, Hasler P, Holzgreve W, Hahn S (2007): Neutrophil NETs: a novel contributor to preeclampsia-associated placental hypoxia? Semin Immunopathol 29: 163-167.

38. Brill A, Fuchs TA, Savchenko AS, et al. (2012): Neutrophil extracellular traps promote deep vein thrombosis in mice. J Thromb Haemost 10: 136-144

39. Cools-Lartigue J, Spicer J, McDonald B, et al. (2013): Neutrophil extracellular traps sequester circulating tumor cells and promote metastasis. J Clin Invest [Epub ahead of print].

40. Kessenbrock K, Krumbholz M, Schonermarck U, et al. (2009): Netting neutrophils in autoimmune small-vessel vasculitis. Nat Med 15: 623-625.

41. Khandpur R, Carmona-Rivera C, Vivekanandan-Giri A, et al. (2013): NETs are a source of citrullinated autoantigens and stimulate inflammatory responses in rheumatoid arthritis. Sci Transl Med 5: 178ra140.

42. Knight JS, Carmona-Rivera C, Kaplan MJ (2012): Proteins derived from neutrophil extracellular traps may serve as self-antigens and mediate organ damage in autoimmune diseases. Front Immunol 3: 380 . 